\title{
Erucismo por Lonomia spp em Teresópolis, RJ, Brasil. Relato de um caso provável e revisão da literatura
}

\author{
Lonomia erucism in Teresópolis, Rio de Janeiro State, Brazil. \\ Report of a probable case and review
}

\author{
Maria Sueli Corrêa ${ }^{1}$, Rodrigo Siqueira-Batista ${ }^{1,2,3}$, Andréia Patrícia Gomes ${ }^{1,2,4}$, Adbell Franco- \\ Barbosa ${ }^{5}$, Ana Candida Arruda Verzola ${ }^{1}$, Fabiana Ribeiro Queiroz de Oliveira ${ }^{6}$, Fabiano Alves \\ Squeff ${ }^{1}$, Joaquim Maurício da Motta-Leal-Filho ${ }^{1}$, Renato Henriques Tavares ${ }^{1}$, Daniela Silva de \\ Amorim ${ }^{1}$, Nelson Luís De-Maria-Moreira ${ }^{7}$ e Sávio Silva Santos ${ }^{1}$
}

\begin{abstract}
RESUM0
Relata-se 0 caso de um homem de 44 anos, natural do Rio de Janeiro, residente no município de Teresópolis ( RJ), vítima de um provável acidente por lagarta do gênero Lonomia, que evoluiu com quadro caracterizado por anemia hemolítica, plaquetopenia e insuficiência renal aguda. 0 diagnóstico de erucismo por Lonomia foi estabelecido a partir da anamnese e das manifestações clínicas e laboratoriais. 0 esquema terapêutico, baseado em hemodiálise e hemotransfusão, resultou em excelente resposta clínica. São discutidos os aspectos clínicos e fisiopatológicos do erucismo por Lonomia.
\end{abstract}

Palavras-chaves: Erucismo. Lonomia spp. Coagulação intravascular disseminada. Insuficiência renal aguda.

\begin{abstract}
This is a case report of a 44-year-old male living in Teresópolis, RJ, Brazil, probably poisoned by contact with a Lonomia caterpillar, who presented hemolytic anemia, decreased platelet count and acute renal insufficiency. Lonomia erucism diagnosis was established by anamnesis and clinical and laboratory manifestations. Therapeutic measures consisting of hemotransfusion and hemodialysis were successful. Physiopathologic and clinical features of erucism by Lonomia are discussed.
\end{abstract}

Key-words: Caterpillars envenomation. Lonomia spp. Disseminated intravascular coagulation. Acute renal failure.

0 acidente determinado por lagartas - erucismo - costuma evoluir apenas com sintomas locais ( hiperemia, dor calor, formação de bolhas e outros) mas, eventualmente, pode ocasionar sérias complicações sistêmicas, com evolução fatal em alguns casos, especialmente nos eventos provocados por Lonomia $\mathrm{spp}^{2913}$. Os casos de acidente por este lepidóptero descritos no Brasil, até 0 presente momento, ocorreram nas Regiões Norte e Sul, não havendo menção a episódios no Estado do Rio de Janeiro. № Sul ${ }^{13}$ vem sendo observado um incremento na ocorrência de erucismo, com relevante incidência -, de 1,1 até 24,6 casos por 100 mil habitantes ${ }^{4}$ principalmente nas áreas rurais ${ }^{1}$. Espécimes de Lonomia produzem um veneno rico em substâncias com atividades procoagulantes e fibrinolíticas - p. ex., a enzima lonofibrase ${ }^{12}$, capazes de desencadear uma síndrome hemorrágica semelhante à coagulação intravascular disseminada (CID) ${ }^{10} 17$, com incremento nos produtos de degradação do fibrinogênio (PDFs) e diminuição sérica do plasminogênio, fibrinogênio

1. Disciplina de Clínica Médica da Faculdade de Medicina de Teresópolis da Fundação Educacional Serra dos Órgãos, Teresópolis, RJ. 2. Centro de Vigilância Epidemiológica da Secretaria Estadual de Saúde, Rio de Janeiro, RJ. 3. Serviço de Clínica Médica do Hospital Universitário Clementino Fraga Filho da Universidade Federal do Rio de Janeiro, Rio de Janeiro, RJ. 4. Disciplina de Doenças Infecciosas e Parasitárias da Faculdade de Medicina de Teresópolis da Fundação Educacional Serra dos Órgãos, Teresópolis, RJ. 5. Serviço de Cirurgia Geral do Hospital das Clínicas de Teresópolis Costantino Ottaviano da Fundação Educacional Serra dos Órgãos, Teresópolis, RJ. 6. Serviço de Pediatria da Hospital dos Servidores do Estado do Rio de Janeiro, Rio de Janeiro, RJ. 7. Serviço de Oftalmologia do Hospital Universitário Antônio Pedro da Universidade Federal Fluminense, Niterói, RJ.

Endereço para correspondência: Prof. Rodrigo Siqueira Batista. Disciplina de Clínica Médica/Fundação Educacional Serra dos Órgãos (FESO). Av. Alberto Torres 111, Alto, 25964-000 Teresópolis, RJ.

Tel: 5521 2641-7000.

e-mail: anaximandro@ hotmail.com

Recebido para publicação em:01/04/2002

Aceito em: 16/6/2004 
e fator XIII ${ }^{59111618}$. Sangramentos e insuficiência renal aguda são as principais manifestações destes desarranjos, permanecendo ainda pouco clara a patogênese desta última alteração $0^{356715}$. A letalidade pode chegar a 2,5\%14.

0 presente artigo apresenta um provável caso de erucismo por Lonomia spp, o qual evoluiu com síndrome hemorrágica e insuficiência renal aguda, compondo suas manifestações em relação ao descrito na literatura.

\section{RELATO DO CASO}

Paciente masculino, branco, 44 anos, natural do Rio de Janeiro, residente em Teresópolis, há dois anos, comerciante, casado.

Queixas principais. Amarelão nos olhos e pele e diarréia com sangue.

História da doença atual. Cinco dias antes da internação 0 paciente estava no jardim de sua residência quando, ao apoiarse em uma árvore, sentiu intensa dor em queimação na palma da mão direita. Neste momento, notou que havia tocado em uma lagarta. 0 animal foi descrito como possuindo mais ou menos $5 \mathrm{~cm}$ de comprimento, com corpo recoberto de cerdas ou espículas pontiagudas ou ramificadas, de coloração marromclaro-esverdeada. Após seis horas do acidente, evoluiu com importante edema no membro superior direito e ocorrência de um pico febril $\left(39^{\circ} \mathrm{C}\right)$, aliviado com dipirona. № dia seguinte, houve melhora da dor e do edema no membro afetado, com surgimento de vômitos ( caracterizado por conteúdo alimentar), diminuição do débito urinário, dor lombar bilateral ( na região da loja renal) irradiada para flancos e que piorava com a deambulação. Procurou assistência médica 48 horas após 0 acidente, sendo realizada ultra-sonografia (USG) abdominal que não mostrou alterações importantes; foi medicado com analgésicos e antieméticos ( sem melhora) e instituída reposição volêmica. 0 paciente, entretanto, manteve-se oligúrico. No dia seguinte apresentou episódios de enterorragia, melena e hematúria macroscópica, evoluindo com perpetuação da oligúria, a despeito da estabilidade hemodinâmica. Dois dias após 0 primeiro atendimento surgiram icterícia (avaliável em mucosas), soluços, novos episódios de enterorragia e melena, tendo sido encaminhado ao Hospital das Clínicas de Teresópolis Costantino Ottaviano (HCTCO), para avaliação.

Na história patológica pregressa, história fisiológica e familiar não foram mencionados dados dignos de registro; na história social, de importante, relatou-se etilismo social e tabagismo (carga tabágica de 20 maços-ano).

Exame físico. 0 paciente apresentava-se estável, lúcido e orientado no tempo eno espaço, hipocorado $(++/ 4+)$, hidratado, ictérico, afebril, sem queixas no momento. Manteve-se sem febre durante 0 período, com níveis pressórios estáveis, diurese protraída, sem evidencias de novo sangramento. Murmúrio vesicular audível, sem ruídos adventícios. Ritmo cardíaco regular com bulhas normofonéticas, sem sopros ou extrassístoles. Sinais vitais: FC 80bpm, TA 160x80mmHg, FR 24irpm, Tax 36,8 $\mathrm{C}$. Abdômen globoso, sem massas ou visceromegalias, peristalse diminuída e presença de hematomas em flancos. Membros superiores também com presença de hematomas.

Avaliação laboratorial. Os exames laboratoriais ( Tabela 1) demonstraram retenção importante de escórias nitrogenadas, acidose metabólica, anemia hemolítica e plaquetopenia.

Evolução. Com base nos achados clínicos e laboratoriais foi considerada a suspeita de acidente por Lonomia. Ao ser apresentada uma fotografia da forma larvar deste lepidóptero ao paciente, este reconheceu- 0 como agressor. Em virtude das alterações encontradas, optou-se por internação hospitalar e tratamento dialítico de urgência. Após introdução do cateter para diálise, 0 doente apresentou hemorragia abundante no local da punção, não impedindo, no entanto, a realização do procedimento dialítico. Foram transfundidos $600 \mathrm{ml}$ de concentrado de hemácias. Quarenta e oito horas após 0 atendimento no HCTCO, o paciente apresentou agravamento dos episódios hemorrágicos, evoluindo com equimoses difusas pelo corpo, principalmente em membros superiores e abdômen. Recebeu, então, dez unidades de concentrados de plaquetas. No dia seguinte, houve melhora dos episódios hemorrágicos, mantendo-se a terapia dialítica, em decorrência da perpetuação do quadro de anúria. Devido aos episódios de hematêmese, foi realizada endoscopia digestiva alta (EDA), neste mesmo dia, não sendo observadas alterações significativas. Cerca de sete dias após a internação no HCTCO houve resolução dos episódios hemorrágicos, mantendo-se 0 tratamento dialítico, em virtude das escórias permanecerem elevadas. Não foi realizada soroterapia específica por indisponibilidade do imunobiológico. 0 paciente recebeu alta hospitalar, no décimo sexto dia de internação. Deste então, permaneceu em acompanhamento ambulatorial com diminuição progressiva dos níveis de escórias nitrogenadas (Tabela 2).

\section{DISCUSSÃ0}

Os acidentes por lagartas do gênero Lonomia vêm se tornando mais freqüentes nos últimos anos, fato provavelmente relacionado ao desequilíbrio ecológico provocado pelos constantes desmatamentos ${ }^{7}$. Uma série de 199 casos mostrou a predominância de ocorrências no sexo masculino (63\%), em jovens ( $45 \%$ de $0-19$ anos) e com lesões nas mãos $(38 \%)^{14}$.

0 presente relato sugere fortemente a ocorrência do primeiro caso de erucismo por Lonomia no Rio de Janeiro. 0 enfermo apresentava, à admissão, plaquetopenia, anemia hemolítica e insuficiência renal aguda, podendo ser estabelecida, durante a obtenção da anamnese, uma importante relação causal entre 0 contato com 0 animal e 0 desenvolvimento das alterações descritas. Tal possibilidade tornou-se ainda mais substancial após o reconhecimento da lagarta pelo enfermo, consoante 0 descrito. De fato, as manifestações identificadas no paciente são bastante compatíveis com 0 diagnóstico proposto ${ }^{8}{ }^{10}$, tal qual 0 amplamente veiculado na literatura. Conforme demonstrado 
Tabela 1 - Evolução dos exames laboratoriais.

\begin{tabular}{|c|c|c|c|c|c|c|c|c|}
\hline \multirow[t]{2}{*}{ Exame } & \multirow[t]{2}{*}{ Valores de referência } & \multirow[t]{2}{*}{ Admisão } & \multicolumn{6}{|c|}{ Dias de Internação } \\
\hline & & & $1^{0}$ & $2^{\circ}$ & $3^{\circ}$ & $4^{\circ}$ & $6^{\circ}$ & $11^{\circ}$ \\
\hline Leucócitos & $5.000-10.000 \mathrm{P} / \mathrm{mm}^{3}$ & 8.700 & 9.700 & - & 10.400 & 10.200 & 14.500 & 15.300 \\
\hline Eosinófilos & $2-5 \%$ & 3 & 3 & - & 0 & 1 & 1 & 1 \\
\hline Mielócitos & $0 \%$ & 0 & 0 & - & 0 & 0 & 0 & 0 \\
\hline Metamielócitos & $0 \%$ & 0 & 0 & - & 0 & 0 & 0 & 0 \\
\hline Bastões & $3-5 \%$ & 3 & 2 & - & 4 & 4 & 2 & 4 \\
\hline Segmentados & $55-66 \%$ & 60 & 54 & - & 47 & 58 & 81 & 58 \\
\hline Linfócitos & $20-35 \%$ & 33 & 37 & - & 45 & 34 & 12 & 33 \\
\hline Monócitos & $4-8 \%$ & 1 & 2 & - & 4 & 3 & 4 & 4 \\
\hline Hemácias & $\mathrm{H} 4,5-5,5 / \mathrm{M} 4,0-5,5$ & 2,19 & 2,11 & - & 2,11 & 2,40 & 2,63 & 2,60 \\
\hline Hematócrito & H $40-50 \% / M 4,0-5,5 \%$ & 19,0 & 18,6 & 20 & 17,5 & 17,0 & 22,2 & 21,8 \\
\hline Hemoglobina & H 12-17g/dl/M 11-14 g/dl & 6,2 & 7,1 & - & 6,3 & 6,3 & 7,7 & 6,7 \\
\hline Plaquetas & $150.000-400.000 / \mathrm{mm}^{3}$ & 83.000 & - & 71.000 & 150.000 & 147.000 & 216.000 & 150.000 \\
\hline Uréia & $15-50 \mathrm{mg} \%$ & 180 & 168 & 116 & 178 & - & 153 & 117 \\
\hline Creatinina & Até $1.5 \mathrm{mg} \%$ & 17 & 15 & 10,7 & 18,7 & - & 15,6 & 13,8 \\
\hline Sódio & $135-150 \mathrm{mEq} / \mathrm{L}$ & - & 127 & - & 129 & - & 133 & 135 \\
\hline Potássio & 3.5-5.5mEq/L & - & 3,5 & 3.8 & 3,7 & - & 4,8 & 4,3 \\
\hline Glicose & $70-110 \mathrm{mg} \%$ & 97 & - & - & - & - & - & - \\
\hline Bilirrubina total & Até $1.2 \mathrm{mg} / 100 \mathrm{ml}$ & 3,0 & 2,5 & - & - & - & - & - \\
\hline TS & Duke Até 3 minutos & $4^{\prime}$ & - & - & $1^{\prime}$ & $1^{\prime} 15^{\prime \prime}$ & $1^{\prime}$ & $2^{\prime}$ \\
\hline TC & Lee/Write 4-8 minutos & $9^{\prime} 06^{\prime \prime}$ & - & - & $7^{\prime}$ & $6^{\prime} 15^{\prime \prime}$ & $6^{\prime} 25^{\prime \prime}$ & $6^{\prime} 59^{\prime \prime}$ \\
\hline TP & $13^{\prime \prime} / 100 \%$ & - & - & $13^{\prime \prime} / 100 \%$ & $13^{\prime \prime} / 100 \%$ & $13^{\prime \prime} / 100 \%$ & $14^{\prime \prime} / 84 \%$ & $14^{\prime \prime} / 83 \%$ \\
\hline PTT & $30-40^{\prime \prime}$ & $36^{\prime \prime}$ & - & $30^{\prime \prime}$ & $36^{\prime \prime}$ & $33^{\prime \prime}$ & $46^{\prime \prime}$ & $43^{\prime \prime}$ \\
\hline Reticulócitos & $0-2 \%$ & 2,5 & - & 7 & - & - & - & - \\
\hline Amilase & $60-120 \mathrm{UI}$ & 263 & 257 & - & - & - & - & - \\
\hline ST & $20-50 \%$ & 35 & - & - & - & - & - & - \\
\hline AST & $0-42 \mathrm{U} / \mathrm{L}$ & 72 & 71 & - & - & - & - & - \\
\hline ALT & Até 40 U/L & 68 & 68 & - & - & - & - & - \\
\hline $\mathrm{LDH}$ & $200-480$ UI & 800 & 690 & - & - & - & - & - \\
\hline
\end{tabular}

ALT = alanina aminotransferase $\mathrm{ST}=$ saturação da transferrina $\mathrm{AST}=$ aspartato aminotransferase $\mathrm{TAP}=$ tempo de protrombina $\mathrm{H}=\mathrm{Homem} /$ $\mathrm{M}=$ Mulher TC = tempo de coagulação $\mathrm{LDH}=$ desidrogenase lática TS = tempo de sangramento PTT = tempo de troboplastina parcial

Tabela 2 - Evolução dos exames laboratoriais-ambulatoriais.

\begin{tabular}{|c|c|c|c|c|c|c|}
\hline \multirow[t]{2}{*}{ Exame } & \multirow[t]{2}{*}{ Valores de referência } & \multicolumn{5}{|c|}{ Dias de internação } \\
\hline & & $17^{\circ}$ & $19^{\circ}$ & $24^{\circ}$ & $33^{\circ}$ & $41^{\circ}$ \\
\hline Leucócitos & $5.000-10.000 / \mathrm{mm}^{3}$ & 7.400 & - & 8.500 & - & 7.800 \\
\hline Basófilos & $0-1 \%$ & 0 & - & 0 & - & 0 \\
\hline Eosinófilos & $2-5 \%$ & 2 & - & 0 & - & 3 \\
\hline Mielócitos & $0 \%$ & 0 & - & 0 & - & 0 \\
\hline Metamielócitos & $0 \%$ & 0 & - & 0 & - & 0 \\
\hline Bastões & $3-5 \%$ & 3 & - & 3 & - & 1 \\
\hline Segmentados & $55-66 \%$ & 65 & - & 63 & - & 58 \\
\hline Linfócitos & $20-35 \%$ & 26 & - & 32 & - & 38 \\
\hline Monócitos & $4-8 \%$ & 4 & - & 1 & - & 0 \\
\hline Hemácias & $\mathrm{H} 4,5-5,5 / \mathrm{M} 4,0-5,5$ & 2,86 & - & 3,1 & - & 3,07 \\
\hline Hematócrito & H 40-50\%/M 4,0-5,5\% & 23,9 & - & 25,0 & 27,8 & 26,4 \\
\hline Hemoglobina & H 12-17g/dl/M 11-14g/dl & 8,8 & - & - & 6,3 & 6,3 \\
\hline Plaquetas & $150.000-400.000 / \mathrm{mm}^{3}$ & - & - & - & - & 240.000 \\
\hline Uréia & $15-50 \mathrm{mg} \%$ & 129 & 96 & 74 & 54 & 69 \\
\hline Creatinina & Até $1,5 \mathrm{mg} \%$ & 5,1 & 4,3 & 2,1 & 2 & 1,7 \\
\hline Sódio & $135-150 \mathrm{mEq} / \mathrm{L}$ & - & - & 134 & - & 131 \\
\hline Potássio & $3,5-5,5 \mathrm{mEq} / \mathrm{L}$ & 4 & 4 & 5,1 & 4 & 3,7 \\
\hline Glicose & $70-110 \mathrm{mg} \%$ & - & - & - & - & 90 \\
\hline Lipídeos totais & $400-800 \mathrm{mg} / \mathrm{d} 1$ & - & - & - & - & 902 \\
\hline Colesterol & $150-250 \mathrm{mg} / \mathrm{dl}$ & - & - & - & - & 211 \\
\hline ST & $20-50 \%$ & - & - & - & - & 36 \\
\hline Ferritina & $32-501$ & - & - & - & - & 142 \\
\hline LDH & 200-480UI & - & - & - & - & 250 \\
\hline Cálcio total & $9-10 \mathrm{mg} / \mathrm{dl}$ & - & - & - & - & 9,4 \\
\hline Ácido úrico & $1,5-7 \mathrm{mg} / \mathrm{dl}$ & - & - & - & - & 5,5 \\
\hline Fósforo & $2,5-4,8 \mathrm{mg} / \mathrm{dl}$ & - & - & - & - & 3,5 \\
\hline Reticulócitos & $0-2 \%$ & - & - & - & - & 3 \\
\hline
\end{tabular}


por Arocha-Piñango ${ }^{1}$, Burdmann e cols ${ }^{2}$, Fraiha e cols ${ }^{6}$ e Seibert e cols ${ }^{15}$, a tríade composta por anemia hemolítica, plaquetopenia e insuficiência renal aguda é bastante encontradiça após 0 contato com Lonomia $\operatorname{spp}^{15815}$. Vale ressaltar que a patogênese deste último distúrbio permanece incerta, estimando-se que haja participação (1) da deposição de microtrombos nos glomérulos, (2) de uma possível ação nefrotóxica do veneno e (3) da hipotensão e isquemia promovidas pelos distúrbios hemorrágicos ${ }^{5}$.

Destacam-se, igualmente, os sinais locais apresentados pelo enfermo, o que está de acordo com as observações de Couppie e cols ${ }^{3}$, os quais relataram cinco casos de envenenamento por Lonomia que cursaram com dor, edema, placas eritematosas e necrose de pele - ainda que estas duas últimas alterações não tenham sido observadas no caso em questão.

Em relação ao tratamento, o paciente recebeu apenas medidas de suporte - cuidados locais, controle do equilíbrio hidroeletrolítico e ácido-básico, hemotransfusão, reposição de fatores da coagulação (fibrinogênio ou crioprecipitado do fator VIII) e terapia dialítica - as quais compõem a conduta na maior parte dos $\operatorname{cas}^{5}{ }^{5}$. São contra-indicados sangue total e plasma fresco, pelo potencial agravamento da coagulação intravascular disseminada relacionada a tais agentes ${ }^{8}$. Habitualmente, há boa resposta terapêutica. Mais recentemente, vem sendo indicado soro antilonômico $(\text { SALon })^{716}$. No entanto, o emprego de SALon tem utilização restrita, estando na fase de estudos clínicas ${ }^{891016 .}$

Considerações finais. 0 caso descrito reitera, uma vez mais, a capital importância da anamnese e do exame físico para 0 estabelecimento do diagnóstico - sem uma história adequadamente obtida dificilmente se pensaria em tal possibilidade. Ademais, fica 0 alerta para a ocorrência de novos casos de erucismo por Lonomia no Estado do Rio de Janeiro, área até então considerada livre desta condição mórbida.

\section{REFERÊNCIAS BIBLIOGRÁFICAS}

1. Arocha-Piñango CL. Fibrinolisis producida por contato com orugas. Acta Cientifica Venezolana 18: 136-137,1967.

2. Burdmann EA, Antunes I, Saldanha LB, Abdulkader RC. Severe acute renal failure induced by the venom of Lonomia caterpillars. Clinical Nephrologic 46: 337-339,1996.

3. Couppie P, Marty C, Sainte-Marie D, Pradinaud R. Poisonous caterpillars in French Guyana. 5 cases. Annals Dermatology Venereology 125: 489491, 1998.
4. Duarte AC, Caovilla JJ, Hermann F, Renner LD. Hemorragia Gengivo-jugal pós-contato com Lonomia obliqua. Revista de Medicina do Hospital São Vicente de Paula 9: 52-54, 1997.

5. Fan HW, Cardoso JL, Olmos RD, Almeida FL, Viana RP, Martinez AP. Hemorrhagic syndrome and acute renal failure in a pregnant woman after contact with Lonomia caterpillars: a case report. Revista Instituto Medicina Tropical São Paulo 40: 119- 120, 1998.

6. Fraiha H, Ballarini AJ, Leão RNQ, Costa JR, Dias LB. Síndrome hemorrágica por contato com lagartas de mariposa (Lepidoptera, Saturniidae). In: Instituto Evandro Chagas: 50 anos de contribuição às ciências biológicas e à medicina tropical. Fundação Serviços de Saúde Pública (Belém) 2: 811-820, 1986.

7. Fraiha H, Costa Júnior D, Leão RNQ. Acidentes por contato com larvas de Lonomia. In: Leão RNQ ( ed) Doenças Infecciosas e Parasitárias: Enfoque Amazônico. Cejup/UEPA/Instituto Evandro Chagas, Belém, p.799-804, 1997.

8. Fundação Nacional de Saúde. Manual de Diagnóstico e Tratamento de Acidentes por Animais Peçonhentos. Ministério da Saúde, Brasília, 2001.

9. Gonçalves MLC, Siqueira-Batista R, Artemenko SR, Gomes AP, Igreja RP. Acidentes por outros animais peçonhentos. In: Siqueira-Batista R, Gomes AP, Igreja RP, Huggins DW ( eds) Medicina Tropical - Abordagem Atual das Doenças Infecciosas e Parasitárias. $1^{\text {a }}$ edição, Cultura Médica, Rio de Janeiro, p. 149-166, 2001.

10. Gonçalves MLC, Vital ML, Gomes AP, Siqueira-Batista R, Andrade E. Acidentes por outros animais de importância médica. In: Siqueira-Batista R, Gomes AP, Santos SS, Almeida LC, Figueiredo CES, Bedoya Pacheco SJ (eds) Manual de Infectologia. $1^{\text {a }}$ ediçãa, Revinter, Rio de Janeiro, p. 515518, 2003.

11. Guerrero B, Arocha-Pinango CL. Activation of human prothrombin by the venom of Lonomia achelous (Cramer) caterpillars. Thrombosis Research 66: 169-177, 1992.

12. Pinto $\mathrm{AF}$, Dobrovolski R, Veiga $\mathrm{AB}$, Guimarães JA. Lonofibrase, a novel alpha-fibrinogenase from Lonomia obliqua caterpillars. Thrombosis Research 113:147-154, 2004.

13. Roodt AR, Salomon OD, Orduna TA. Accidents due to Lepidoptera with special reference to Lonomia sp. Medicina (Buenos Aires) 60: 964-972, 2000.

14. Rubio GBG. Vigilância epidemiológica da distribuição da lagarta Lonomia obliqua Walker, 1885, no Estado do Paraná, Brasil. Cadernos de Saúde Pública 17:1036, 2001.

15. Seibert CS, Shinohara EM, Sano-Martins IS. In vitro hemolytic activity of Lonomia obliqua caterpillar bristle extract on human and Wistar rat erythrocytes. Toxicon 41:831-839, 2003

16. Silva WD, Campos CM, Gonçalves LR, Souza-e-Silva MC, Higashi HG, Yamagushi IK, Kelen EM. Development of an antivenom against toxins of Lonomia obliqua caterpillars. Toxicon 34: 1045-1049, 1996.

17. Veiga $\mathrm{AB}$, Pinto $\mathrm{AF}$, Guimarães JA. Fibrinogenolytic and procoagulant activities in the hemorrhagic syndrome caused by Lonomia obliqua caterpillars. Thrombosis Research 111:95-101, 2003.

18. Zannin M, Lourenço DM, Motta G, Dalla Costa LR, Grando M, Gamborgi GP, Noguti MA, Chudzinski-Tavassi AM. Blood coagulation and fibrinolytic factors in 105 patients with hemorrhagic syndrome caused by accidental contact with Lonomia obliqua caterpillar in Santa Catarina, southern Brazil. Thrombosis Haemostasis 89:355-364, 2003. 